\title{
Monolithic Silicon Probes with Flexible Parylene Cables for Neural Prostheses
}

\author{
Changlin Pang ${ }^{1 *}$, Jorge G. Cham ${ }^{2}$, Sam Musallam ${ }^{3}$, Yu-Chong Tai ${ }^{1}$, Joel W. Burdick ${ }^{2}$, and Richard A. Andersen ${ }^{3}$ \\ ${ }^{1}$ Caltech Micromachining Lab, California Institute of Technology, Pasadena, CA 91125, USA \\ ${ }^{2}$ Department of Mechanical Engineering, California Institute of Technology, Pasadena, CA 91125, USA \\ ${ }^{3}$ Division of Biology, California Institute of Technology, Pasadena, CA 91125, USA
}

\begin{abstract}
This work presents the first parylene-insulated silicon probes, which are used for neural prostheses to record high-level cognitive neural signals. With parylene technology, our probes have several advantages compared with the current devices. First, instead of inorganic materials (e.g. silicon dioxide, silicon nitride), the electrodes and conduction traces on the probes are insulated by parylene, an easily-deposited polymer with mechanical flexibility and biocompatibility. As a result, the probes exhibit better electrical and mechanical properties. Second, flexible parylene cables are monolithically integrated with the probes, which arm the probes with very high flexibility to be easily assembled to a high density 3-D array and at the same time provide an ideal method to transmit neural signals through skull during chronic recording. The all dry fabrication process and a 4 $\times 4$ probe array (64 electrodes) were demonstrated. The probes were successfully tested electrically and mechanically in rat cortex. Neural signals were properly recorded.
\end{abstract}

Keywords-neural probes; flexible parylene cables; neural prostheses

\section{INTRODUCTION}

An important common goal, although futuristic, is to achieve cortex prostheses using implanted probes to control robotics by pure thoughts (Fig. 1). The first beneficiaries are likely to be patients with spinal-cord injuries, peripheral nerve disease, or amyotrophic lateral sclerosis [1]. To achieve this, 3$\mathrm{D}$ neural probe is needed to record cortex cognitive signals. Various researchers (including the Twente, Washington, Utah and Michigan) have attempted this must-have device for decades but current 3-D neural probe is still far from being satisfactory.

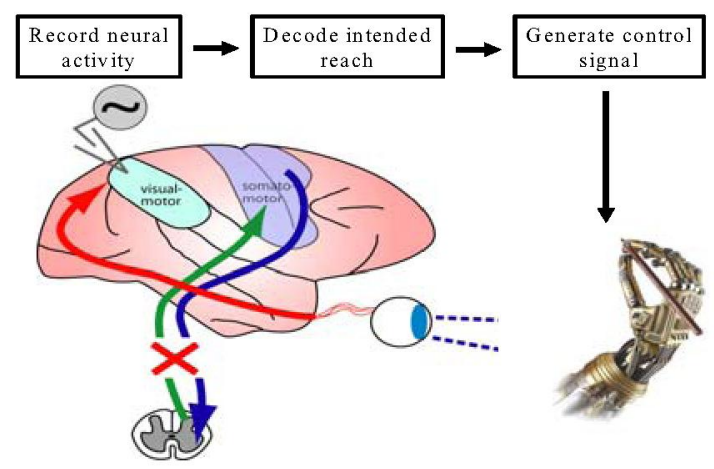

Figure 1. Schematic of the pathway of information flow for the cognitivebased neural prosthetic paradigm.
Ideally, a 3-D multielectrode neuroprobe should have integrated electronics for high $\mathrm{S} / \mathrm{N}$ and flexible cables for through-skull interconnection. Unfortunately, there are two major problems with the current devices. The first one is related to insulating/protecting materials. It's granted that probes have to use silicon when IC is necessary. The question is about insulating materials such as $\mathrm{SiO}_{2}$ and $\mathrm{Si}_{3} \mathrm{~N}_{4}$, which are all subjective to body-fluid corrosion. Worse yet, they are brittle and have reliability issues under stress. The second problem is related to through-skull interconnect. Signals obtained by probes have to be cabled out of skull. Even with telemetry, a cable is still needed to link the in-cortex probes to a telemetry platform that can only be safely mounted under the skull. Cables are important [2]. For example, the Michigan group made the famous silicon cables [3], but their cables are unfortunately subjective to fracture failure. Low yield is especially reported by the authors for longer cables. This work first presents a new method to make 3-D silicon probes enabled by parylene technology. Instead of common inorganic materials (e.g., $\mathrm{SiO}_{2}, \mathrm{Si}_{3} \mathrm{~N}_{4}$ ), our electrodes are insulated completely by parylene, an easily-deposited polymer with mechanical flexibility and biocompatibility. More importantly, this new probe design allows integration of monolithic flexible cables by DRIE process. Better yet, the flexible cables enable high density 3-D arrays for chronic implantation. As a result, this work reports the first parylene-insulated silicon probes, the validation of the use of probes in rat cortex, and the make of a 3-D probe array (64 electrodes).

\section{DEVICES DESIGN}

The geometric design of the probes is shown in Fig. 2. Four shafts with four Pt electrode sites each protrude from a thicker plate. The target shaft thickness is $50 \mu \mathrm{m}$; the shaft width is $75 \mu \mathrm{m}$ at the bottom and $45 \mu \mathrm{m}$ at the outmost section. The lateral taper angle of the chisel-shaped tip is designed to $10^{\circ}$. The width of the trace lines at the outmost shaft section is $2 \mu \mathrm{m}$. Different shaft length is designed for different testing purposes. $8 \mathrm{~mm}$ is for the acute recording of monkey neural signals, $4 \mathrm{~mm}$ and $2 \mathrm{~mm}$ long are for rats. The recording electrode sites are $10 \mu \mathrm{m} \times 10 \mu \mathrm{m}$ and are distributed with a $100 \mu \mathrm{m}$ pitch. The distance between the two shafts are $400 \mu \mathrm{m}$, therefore, a 16-site 2-D electrode array is formed on one probe plate. Several plates can be stacked together to make a 3-D electrode array (Fig. 3(a)). We also designed $2 \mathrm{~mm}$ long probes with integrated parylene cables to do the chronic implantation recording. The parylene cables are monolithically integrated with the probes, and are basically a 'sandwich' structure, which
This work was supported by the National Institutes of Health and the Defense Advanced Research Projects Agency.

* Contacting Author: Changlin Pang is with the Caltech Micromachining Lab, California Institute of Technology, Pasadena, CA 91125, USA. (phone: +1-626-395-2254; fox: +1-626-584-9104; email:changlin@its.caltech.edu). 
has two parylene layers and metal traces between them. 16 parallel $\mathrm{Cr} / \mathrm{Au}$ trace lines are inside a parylene cable for the connection of the 16 electrodes on one probe plate. With the flexible parylene cables, we can easily implant the electrode array for chronic recording. Fig. 3(b) shows the whole design of implantation setup for chronic recording.

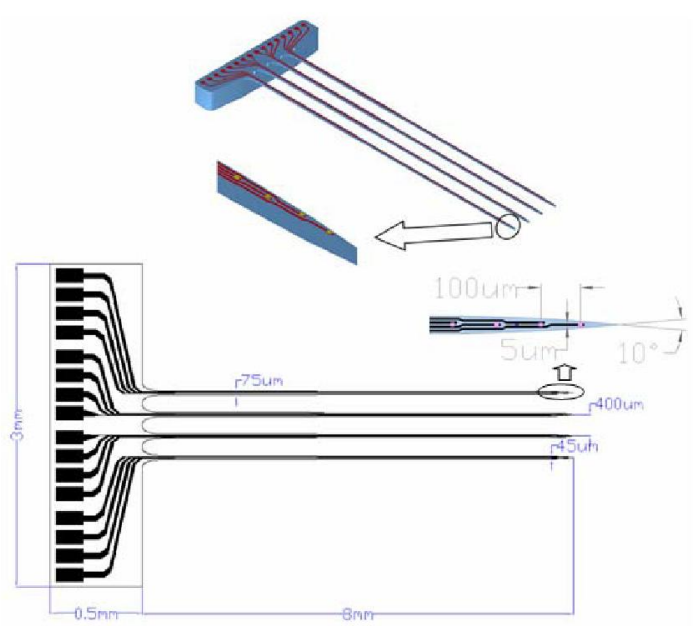

Figure 2. Geometric design of the probes.

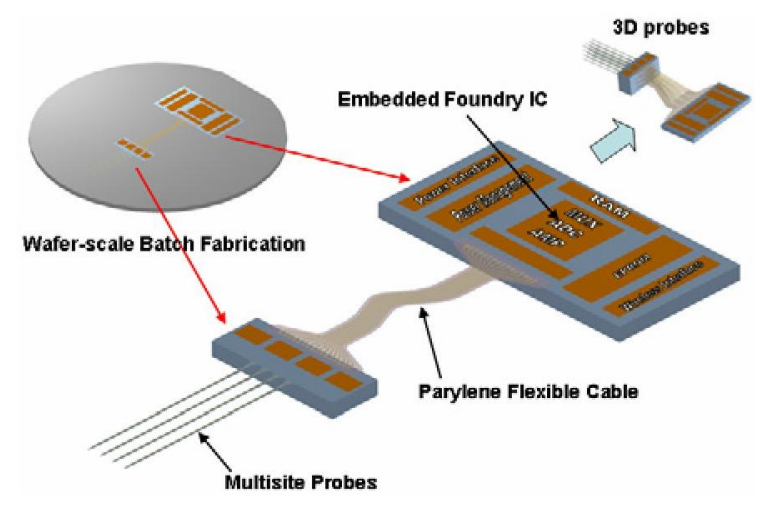

(a)

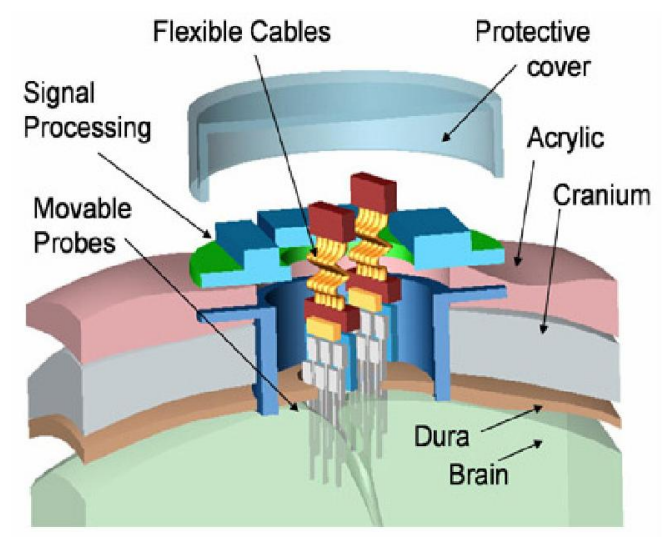

(b)

Figure 3. (a) Concept of parylene flexible cables and method to make 3-D probes array with parylene cables connected to connection and control circuit; (b) Schematic of cortical implantation using parylene cables.

\section{FABRICATION PROCESS}

The silicon probe and parylene cable fabrication process flow is shown in Fig. 4, which is based on dry etching technology. Lift-off parylene skin technique is used to fabricate parylene cables monolithically. Instead of silicon-on-insulator (SOI) wafers, double-side-polished (DSP) wafers are used, which are less expensive. (a) $\mathrm{XeF}_{2}$ etching is performed on the probes top silicon surface to enhance the adhesion property between silicon and parylene. (b) A photoresist sacrificial layer is patterned on the place where the parylene cables will be. (c) A $1.5 \mu \mathrm{m}$ parylene $\mathrm{C}$ insulating layer is conformably deposited. (d) $\mathrm{Cr} / \mathrm{Au}(\sim 100 \AA / 2000 \AA)$ is thermal evaporated and patterned by lift-off to form the conduction traces. (e) A second parylene C layer $(\sim 1.5 \mu \mathrm{m})$ is deposited as a protective layer; the electrode sites and bonding pads are opened by plasma etching. (f) Ti/Pt $(\sim 200 \AA / 2000 \AA)$ is e-beam evaporated and patterned by lift-off to form the electrode sites. (g) The parylene layers are patterned along the probe shape by plasma etching. (h) Front side DRIE (deep reactive ion etching) ( $\sim 100$ $\mu \mathrm{m}$ deep) is performed to define the probe shape into silicon. (i) Back side DRIE defines the probe thickness, and releases the probes. During backside DRIE etching, the probes are protected by baked photoresist. By depositing protection photoresist on the backside of the finished probes die by die, the probe thickness can be well controlled in $5 \mu \mathrm{m}$ range on whole wafer. (j) Release all the mask and sacrificial photoresist. (k) Break the thin silicon film underneath the parylene cables to release them; break the connection parts between the wafer and the probe plates to release the whole devices.

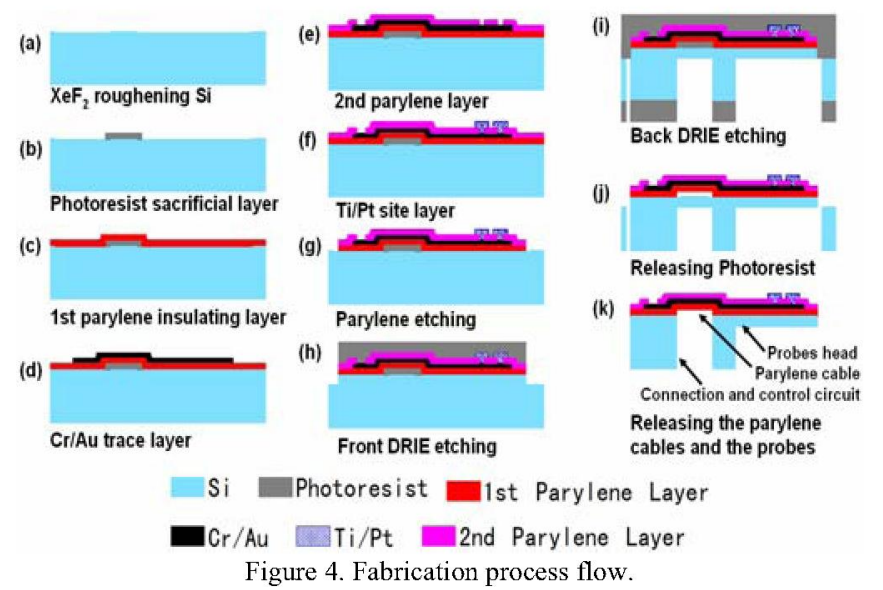

IV. EXPERIMENTAL RESULTS

Optical and SEM pictures of the fabricated probes are shown in Fig. 5. Parylene layer shows very good adhesion property on roughened silicon substrate. No visible delamination or adhesion defect is found between the first parylene layer and the probe silicon substrate, and between the two parylene layers. The probes were tested mechanically and electrically on rat cortex. Fig. 6 shows the electrical and mechanical packaging of the probes for rat cortex acute recording. The probes can be easily inserted into brain tissues without buckle or cracking. Electrode impedance is around 1.5 $\mathrm{M} \Omega$ at $1 \mathrm{kHz}$. Neural signals were properly recorded. Fig. 7 shows the sample filtered neural data recorded from one 
channel of the neural probe in rat cortex; and the sample action potential waveforms.
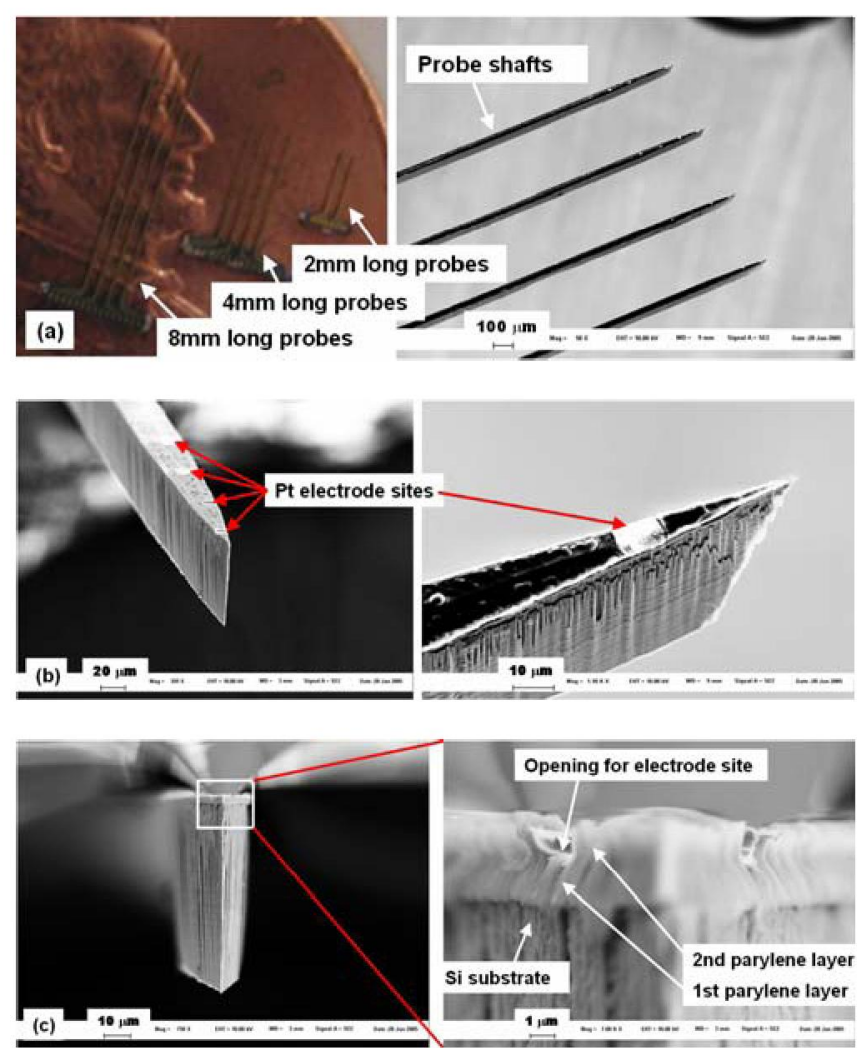

Figure 5. Optical and SEM pictures of the fabricated probes. (a) Pictures of the whole probes and shafts; (b) SEM pictures of the probe tip with multiple electrode sites; (c) SEM pictures of front side view of the probe tip, showing interface between parylene layer and Si substrate and interface between two parylene layers.

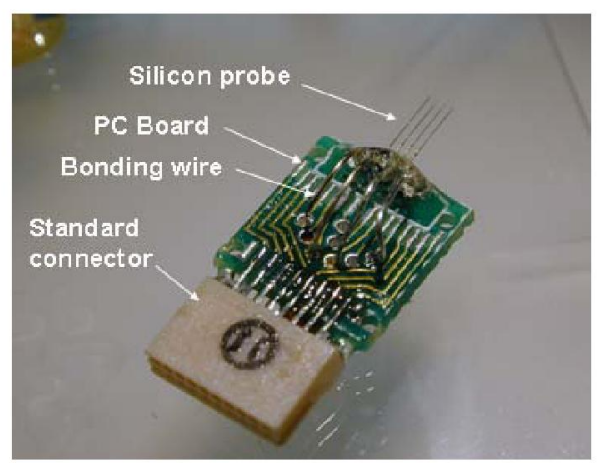

Figure 6. Electrical and mechanical Packaging of the probes for rat cortex acute recording.

The parylene cable is shown in Fig. 8 . It is $3.2 \mathrm{~mm}$ long, $700 \mu \mathrm{m}$ wide and has 16 parallel $\mathrm{Cr} / \mathrm{Au}$ trace lines $(20 \mu \mathrm{m}$ wide) inside. Longer cables and more trace lines can be made using the same method. The parylene cable is monolithically fabricated with a probe array and a connection and control circuit part, and links the two parts together mechanically and electrically. The parylene cable is very flexible, you can even bend it to a sharp angle. The 3-D probe arrays have been demonstrated by easily stacking the 2 -D probe arrays which have monolithic parylene flexible cables (Fig. (a)(b)). Fig. 8(c) shows a $4 \times 23$-D probe array with 32 electrodes and Fig. $8(d)$ shows a $4 \times 43$-D probe array with 64 electrodes.

(a)

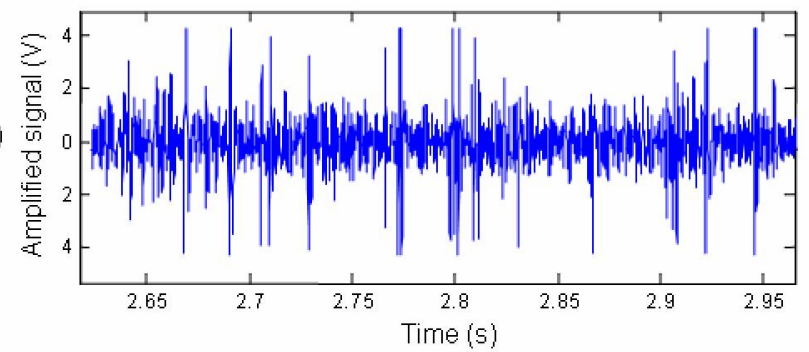

(b)

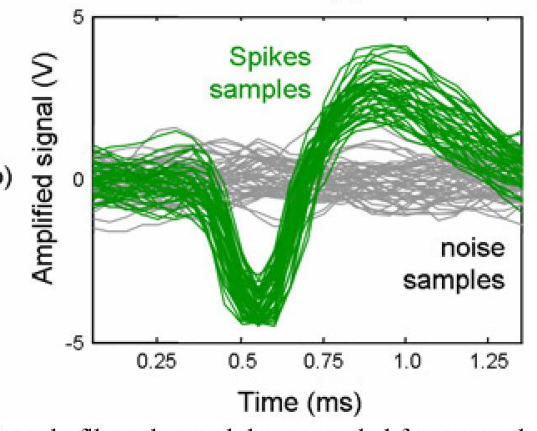

Figure 7: (a) Sample filtered neural data recorded from one channel of the neural probe in rat cortex; (b) Sample action potential waveforms.

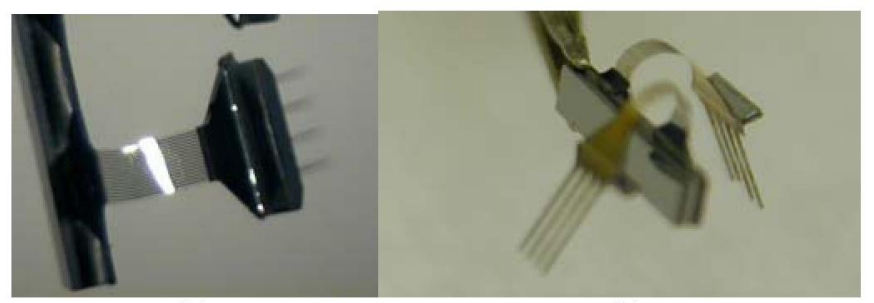

(a)

(b)

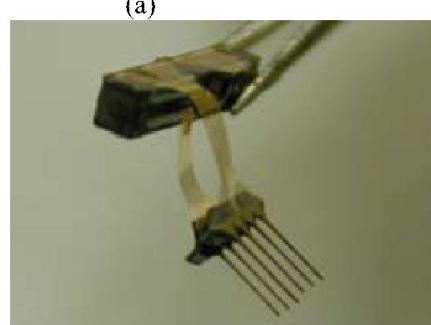

(c)

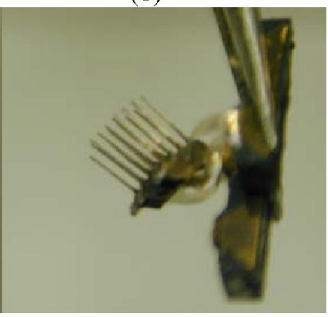

(d)
Figure 8. (a) (b) 2-D probes arrays with integrated flexible parylene cables; (c) 3-D probe arrays $(4 \times 2)$ stacked by two $2-D$ probes plates; (d) 3-D probe arrays $(4 \times 4)$ stacked by four $2-\mathrm{D}$ probes plates.

\section{CONCLUSION}

In conclusion, the new parylene-embedded probes are developed and validated by animal tests, the 3-D probes are made, and the chronic use of the 3-D array in rats and monkeys is underway. The parylene-cabled probes provide the improvement for the ease of fabrication, use and assembly.

\section{ACKNOWLEDGMENT}

We would like to thank Mr. Trevor Roper for assistance with fabrication and the members of the Anderson lab at Caltech for help on vivo testing. 


\section{REFERENCES}

[1] Musallam, S., et al., Cognitive Control Signals for Neural Prosthetics. Science, 2004. 305(5681): p. 258-262.

[2] Norlin, P., et al., A 32-site neural recording probe fabricated by DRIE of SOI substrates. Journal of Micromechanics and Microengineering, 2002. 12: p. 414-419.
[3] Wise, K.D., et al., Wireless implantable microsystems: high-density electronic interfaces to the nervous system. Proceedings of the IEEE, 2004. 92(1): p. 76. 